\title{
Expression du mouvement et pathologie du langage : Perspective typologique en aphasie
}

\author{
Efstathia Soroli \\ UMR 7023, Structures Formelles du Langage, CNRS \& Université de Paris 8 \\ eva.soroli@sfl.cnrs.fr \\ Maya Hickmann \\ UMR 7023, Structures Formelles du Langage, CNRS \& Université de Paris 8 \\ maya.hickmann@slf.cnrs.fr \\ Thi Mai Tran \\ UMR 8163, Savoirs, Textes, Langage, Université Lille Nord de France \\ thimai.tran@univ-lille2.fr \\ Jean-Luc Nespoulous \\ Laboratoire Jacques Lordat, Université de Toulouse Le Mirail \\ jean-luc.nespoulous@univ-tlse2.fr \\ Hélène Boudre \\ Institut d'Orthophonie, Faculté de Médecine, Université Lille Nord de France
}

\section{Introduction}

Des études antérieures en linguistique et en psycholinguistique montrent qu'il existe des variations considérables entre les langues quant à la grammaticalisation et à la lexicalisation des informations concernant l'espace (Talmy, 2000) et que cette variabilité linguistique a un impact sur les représentations des locuteurs (Choi \& Bowerman, 1991 ; Hickmann et al., 2008, 2009 ; Slobin, 2004). Cependant, très peu d'études se sont intéressées à l'impact éventuel de cette répartition dans les troubles acquis du langage du type aphasie, malgré un intérêt croissant pour une perspective translinguistique dans ce domaine (Menn \& Obler, 1990 ; Goodglass \& Gleason, 1991 ; Nespoulous, 1999). Nous proposons dans ce travail d'étudier les performances langagières de deux patients aphasiques, l'un anomique et l'autre agrammatique, dans deux tâches, l'une de compréhension et l'autre de production, à partir de vidéos ou d'animations mettant en scène des déplacements volontaires et provoqués. Nous nous intéresserons plus particulièrement à trois types d'informations (composantes) : la trajectoire, la manière de se déplacer et la cause d'un mouvement provoqué. Nous essaierons de voir premièrement dans quelle mesure les réponses de ces patients peuvent être analysées en référence à la structure de la langue maternelle du locuteur (ici le français), à partir du nombre (densité) et des types de composantes exprimées (focus), ainsi que des moyens utilisés pour les exprimer (locus). Deuxièmement, nous nous intéressons à définir la nature du déficit linguistique (lexical versus syntaxique) dont souffre le patient aphasique et ainsi ouvrir de nouvelles perspectives sur ses éventuelles stratégies compensatoires.

Dans un premier temps, nous ferons quelques rappels sur la façon dont la langue française structure et organise différentes informations relatives au mouvement. Nous rappellerons également les dissociations observables dans la pathologie aphasique au niveau de l'atteinte structurale avec les troubles touchant le lexique et ceux touchant la syntaxe correspondant aux tableaux cliniques des deux patients étudiés (anomie versus agrammatisme). Nous présenterons ensuite le protocole expérimental, proposé à quatorze participants francophones monolingues et réalisés par les deux patients aphasiques de notre étude. Nous analyserons les résultats obtenus par ces deux patients en les comparant à ceux des participants contrôles : nous essaierons ainsi de voir si les patients procèdent ou non à la même répartition de l'information 
spatiale et s'ils maintiennent les mêmes priorités sémantiques dans l'expression du déplacement que les locuteurs francophones sans troubles.

\section{Expression du mouvement et aphasie}

La production d'énoncés est une activité cognitive complexe au cours de laquelle sont mis en jeu de nombreux processus comme la récupération de représentations linguistiques de différentes natures (phonologiques, sémantiques, morphologiques, syntaxiques...), l'agencement de celles-ci conformément aux règles de la langue, la planification et la production de la proposition verbale (Fayol, 1997 ; Segui \& Ferrand, 2000). Cette activité implique pour le locuteur de procéder à une série de choix : choix des informations à transmettre, choix des mots et des structures grammaticales qui vont être utilisées dans le message verbal. Selon l'approche relativiste, la sélection des informations dépend également de la langue parlée par le locuteur. Pour certains auteurs comme Levinson (1996), la langue fonctionne comme un filtre d'informations. Les recherches en typologie sémantique (Lemmens, 2005) montrent que les langues peuvent exprimer de manières différentes des concepts similaires en focalisant sur certaines informations plutôt que d'autres. L'expression du mouvement illustre particulièrement bien la variété systémique des langues et a fait l'objet de nombreux travaux dans le cadre de recherches translinguistiques, dont des travaux sur l'acquisition du langage (Hickmann, 2006; Bowerman, 2007; Choi \& Bowerman, 1991 ; Kopecka, 2004 ; Slobin 1996). On constate en effet, dans le domaine de l'espace, que les langues utilisent des procédures très différentes pour exprimer la localisation ou le déplacement. En ce qui concerne les informations relatives au déplacement comme la trajectoire, la manière et la cause, Talmy $(1985,2000)$ différencie les langues qui lexicalisent ces informations de celles qui les grammaticalisent. Selon cet auteur, les langues fonctionnent de manière relativement équivalentes et présentent des structures assez semblables quand il s'agit d'exprimer un déplacement sans changement de localisation, la manière étant par exemple exprimée dans le verbe principal (ex. 1 et 2) :

(1) Il court dans le salon

Manière (verbe)

(2) He is running in the living-room

Manière (verbe)

En revanche, en cas de changement de localisation, il distingue deux types de langues: les langues $\grave{a}$ cadrage verbal (telles les langues romanes, ex. 3 et 4 français), où la trajectoire est exprimée en priorité par le verbe principal et où la manière, si elle est exprimée, l'est à la périphérie de l'énoncé (par exemple par un gérondif, une expression adverbiale ou une subordonnée : ex. 3 et 4); les langues à satellites (telles les langues germaniques, ex. 5 et 6 anglais et allemand), où la manière est exprimée par le verbe principal et la trajectoire est exprimée à la périphérie du verbe (par exemple par des particules, des prépositions ou des expressions adverbiales : ex. 5 et 6 ).

(3) Il traverse... il monte... il sort

Trajectoire (verbe)

(4) Il sort du salon en courant.

Trajectoire (verbe) Manière (satellite)

(5) He runs out of the living-room.

Manière (verbe) Trajectoire (satellite)

(6) Er rennt über die Strasse. 


\section{Manière (verbe) Trajectoire (satellite)}

Le français fait donc partie, selon Talmy, des langues à cadrage verbal qui privilégient et lexicalisent la trajectoire contrairement aux langues à satellites verbaux qui ont recours à des procédures grammaticales pour exprimer cette information (utilisation de satellites, particules ou prépositions). On notera également qu'en français, lorsque le mouvement est provoqué, la notion de la causalité, est, comme pour la manière, exprimée en périphérie lorsque l'énoncé implique également l'expression de la trajectoire (ex. 7 et 8 ).

(7) Il traverse la route en poussant son caddie.

Trajectoire (verbe) Causalité (satellite)

(8) Il traverse la route en poussant un ballon qui roule.

Trajectoire (verbe) Causalité (satellite) Manière (satellite)

D'autres auteurs se sont intéressés à la possibilité, selon les langues, d'exprimer conjointement plusieurs informations. Les études de Hickmann (2003, 2006), Hendriks \& Hickmann (2006), Hendriks, Hickmann, Demagny (2008), Slobin (2006) ont montré qu'il était plus facile pour un locuteur (adulte ou enfant) anglophone d'exprimer conjointement la trajectoire et la manière que pour un locuteur francophone (ex. 9 et 10) :

(9) He rolled the ball into the kitchen.

Manière (verbe) Trajectoire (satellite)

(10) Il a fait entrer la balle dans la cuisine en la faisant rouler.

Trajectoire (verbe)

Manière (satellite)

L'exemple (10) montre, que l'expression conjointe de la trajectoire et de la manière est possible en français mais au prix d'un allongement de l'énoncé et/ou du recours à une structure syntaxique plus complexe. Pour Slobin (2006), ce coût cognitif supplémentaire explique le fait que les locuteurs francophones préfèrent produire deux énoncés simples lorsqu'ils veulent à la fois exprimer la trajectoire et la manière et qu'ils ont tendance à moins souvent mentionner la manière. Le fait que la langue anglaise permette d'exprimer conjointement plusieurs types d'informations sur le plan structurel expliquerait ainsi pourquoi la densité des composantes exprimées dans les énoncés anglais est systématiquement plus importante par rapport aux énoncés français.

Partant de l'hypothèse relativiste que l'organisation systémique des langues influence la sélection et la répartition des informations spatiales dans le discours, nous pouvons nous interroger, dans le cas des pathologies du langage acquises, sur le rôle du type d'atteinte linguistique (lexicale versus syntaxique) dans l'expression du mouvement en français. Bien que le langage soit le plus souvent atteint à des multiples niveaux en aphasie, la littérature aphasiologique a permis de mettre en évidence des atteintes touchant de manière sélective les processus de lexicalisation (comme dans l'aphasie anomique) ou de façon prédominante les processus de grammaticalisation (comme dans l'agrammatisme dans le cadre sémiologique de l'aphasie de Broca). Des dissociations entre lexique et syntaxe sont ainsi décrites (Kremin, 1994 ; Pillon \& Nespoulous, 1994). En effet, certains patients aphasiques peuvent éprouver des difficultés à trouver les mots sans pour autant rencontrer des difficultés à produire des phrases. D'autres rencontrent des difficultés syntaxiques particulières avec une incapacité ou une difficulté à agencer les mots entre eux et à construire des phrases. On peut ainsi se demander si l'aphasique francophone anomique serait plus pénalisé dans l'expression de la trajectoire et pourrait avoir recours à des éléments grammaticalisés pour compenser son déficit lexical alors que l'aphasique francophone agrammatique rencontrerait davantage de difficultés dans l'expression de la manière ou de la causalité et pourrait, quant 
à lui, utiliser des stratégies lexicales pour pallier ses déficits syntaxiques. Nous faisons l'hypothèse que l'aphasie affecte l'expression du mouvement en modifiant la quantité et la répartition des informations sémantiques ainsi que les modalités d'expression de ces informations (lexicalisation versus grammaticalisation). Nous essaierons de voir dans quelle mesure ces modifications peuvent être mises en rapport avec la langue et / ou avec le déficit linguistique.

\section{Matériel}

Les épreuves proposées à nos participants sont extraites des protocoles expérimentaux élaborés dans le projet de recherche de Hickmann \& Nespoulous intitulé Syntaxe, lexique et représentation de l'espace: perspectives translinguistiques sur l'aphasie, dont est issue la thèse de doctorat de Soroli (en préparation) intitulée: Langage et Cognition spatiale en français, en anglais et en grec: perspectives translinguistiques en aphasie.METTRE DANS LA BIBLIO Les deux épreuves proposées permettent d'étudier respectivement la production et la compréhension d'énoncés faisant intervenir des verbes de mouvements.

\section{Epreuve de production}

L'épreuve de production vise à évaluer l'expression des déplacements volontaires et provoqués. Dans cette épreuve, il est demandé au patient de décrire oralement ce qu'il voit se dérouler dans les petits films correspondant à des vidéos ou à des animations. Ces petits films muets en noir et blanc, d'une durée moyenne de 5 secondes, lui sont présentés sur l'écran d'un ordinateur l'un après l'autre. Une description immédiate de chaque scène est demandée. Les diverses situations proposées correspondent à des déplacements volontaires ou provoqués comprenant différentes trajectoires, manières, ainsi qu'une cause pour les déplacements provoqués.

En ce qui concerne les mouvements volontaires, les actions retenues dans le protocole impliquent différentes Manières $(M)$ invitant l'utilisation de verbes de manière avec ou sans instrument (« faire de la trottinette », «faire du vélo» et «faire des rollers» versus « courir», «sauter», «marcher à quatre pattes »). Le verbe «marcher » a aussi été retenu comme verbe de manière plus neutre que les précédents (M0). Les actions retenues impliquent également différentes Trajectoires (T) soit verticales ( et « descendre »), soit bornées par une ou deux frontières (« entrer» et «sortir » versus « traverser»). Pour ce qui concerne les mouvements provoqués, la causalité (C) était présente dans les animations, correspondant partout à deux actions différentes qui faisaient intervenir différentes manières de provoquer le déplacement pouvant être exprimées par les verbes " pousser » et «tirer » qu'un personnage effectue sur des objets divers (ex. : table, bouée, valise, paquet...). Les types de Manière (M) retenus pour le déplacement de l'objet sont au nombre de deux («glisser» et « rouler»), ceux de Trajectoire (T) étant au nombre de quatre ( « entrer », « traverser », « monter » et « descendre »). Dans ces animations, l'agent se déplace toujours en marchant.

Les lieux où s'effectuent les déplacements sont variés (colline, chemin, porte extérieure et porte dans une pièce vide pour les vidéos ; maison, grotte, dune, rue, rivière, chemin de fer pour les animations). Nous parlerons pour ces items d'items "situés » par opposition aux items non situés qui ne comportent pas d'informations de nature à indiquer une trajectoire (le mouvement se déroulant sur un fond neutre), mettant ainsi maximalement en valeur la manière du déplacement.

L'épreuve comprend 51 items se répartissant en 2 items d'entraînement (1 pour les items situés et 1 pour les items non situés), 36 items situés, 8 items non situés et 5 items distracteurs que nous allons illustrer par quelques exemples.

Les exemples 1 à 3 figurent parmi les items situés, qui concernent les mouvements volontaires dans lesquels, pour l'exemple 1 et 2 , trajectoire $(\mathrm{T})$ et manière $(\mathrm{M})$ de se déplacer sont bien saillantes et pour l'exemple 3 , la manière est plus neutre. 


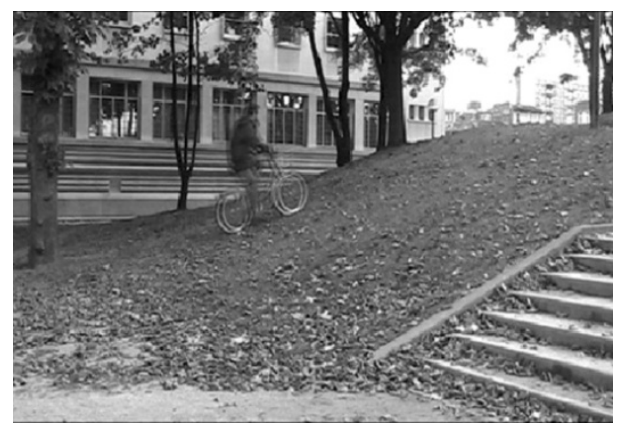

Exemple 1 : monter (T)/faire du vélo $(\mathrm{M})$

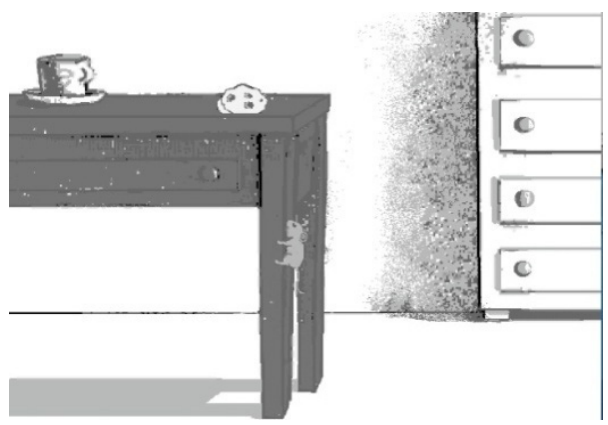

Exemple 2 : descendre (T)/glisser (M)

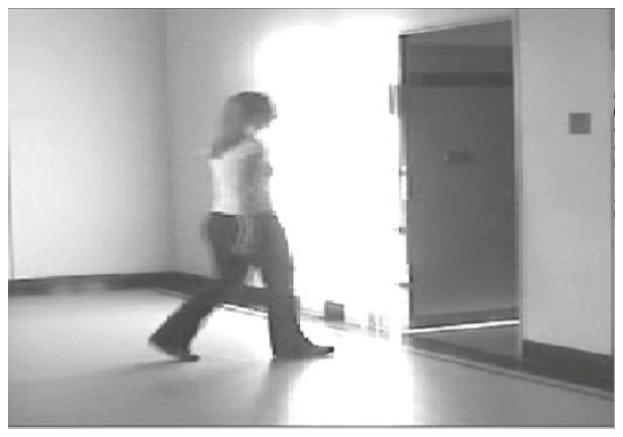

Exemple 3 : entrer (T) /marcher (M0)

L'exemple 4 illustre les mouvements provoqués. Dans cette animation, l'action effectuée par le personnage provoque un déplacement d'objet: l'item fait ainsi intervenir l'expression conjointe de la cause $(\mathrm{C})$, de la manière de cette cause $(\mathrm{Mc})$, de la trajectoire $(\mathrm{T})$, de la manière de déplacement de l'objet (Mo) et de l'agent (Ma).

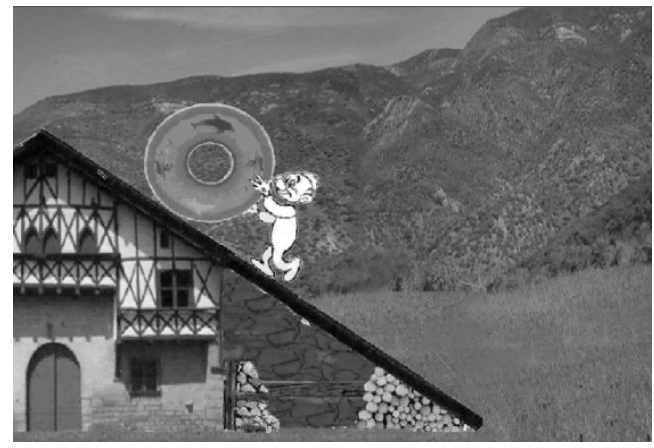

Exemple 4 : pousser (C)/monter (T)/rouler (Mo)/marcher(Ma)

Les exemples 5 et 6 illustrent les items non situés. Ceux-ci visent à induire la seule expression de la manière du déplacement volontaire, notamment par le verbe, quelle que soit la langue. Dans ces animations, les personnages se déplacent d'une certaine façon sur un fond d'écran neutre (impliquant donc une localisation générale indéterminée sans changement de localisation). L'absence de décor ne permet pas d'induire une trajectoire et maximise donc la saillance de la manière $(\mathrm{M})$. Nous parlerons dans ce cas d'items « non situés ». 


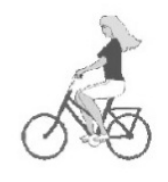

Exemple 5 : rouler à vélo $(\mathrm{M})$

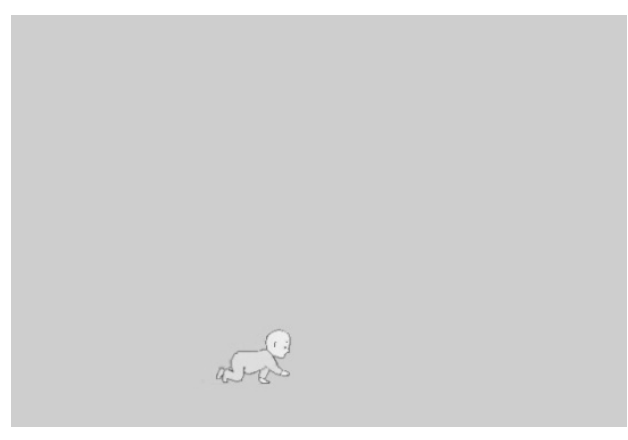

Exemple $6:$ marcher à quatre pattes $(\mathrm{M})$

Enfin, l'exemple 7 correspond à un des 5 items distracteurs montrant des animations faisant intervenir des agents inanimés (balles, paquets...) se déplaçant (mouvement volontaire) et/ou provoquant le déplacement d'autres agents inanimés (mouvement provoqué). Ces items distracteurs ont été introduits afin de faire varier le matériel expérimental et d'éviter que les participants adoptent des stratégies fixes de réponses.

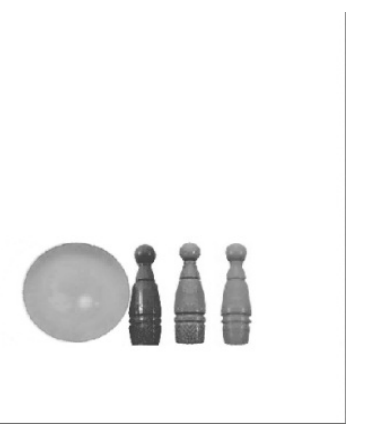

Exemple 7 : La boule roule et renverse les quilles

La consigne indiquée au participant est la suivante: «Vous allez voir une série de vidéos et des animations. Vous devrez juste décrire ce qui se passe dans chaque film. Pour cela, imaginez que vous racontez à quelqu'un qui n'a pas vu le film et ne sait pas ce qui se passe sur la vidéo ».

\section{Epreuve de compréhension}

L'épreuve de compréhension comprend 75 items (dont 1 item d'entrainement, 66 items situés et 8 items distracteurs). Au cours de cette tâche, le participant entend une phrase, et voit en même temps deux vidéos présentées à l'écran simultanément et ne faisant appel qu'à des mouvements volontaires. Il doit alors sélectionner, parmi ces deux vidéos, celle qui correspond à la phrase entendue (une seule réponse correcte). Parmi les 66 phrases cibles, certaines expriment la manière $(\mathrm{M})$ ou la trajectoire $(\mathrm{T})$ seules (ex. : « on voit quelqu'un qui saute» $(\mathrm{M})$, « on voit quelqu'un qui traverse » $(\mathrm{T})$ ), d'autres expriment conjointement la manière et la trajectoire (« on voit quelqu'un qui monte en marchant » : exemple 8). 

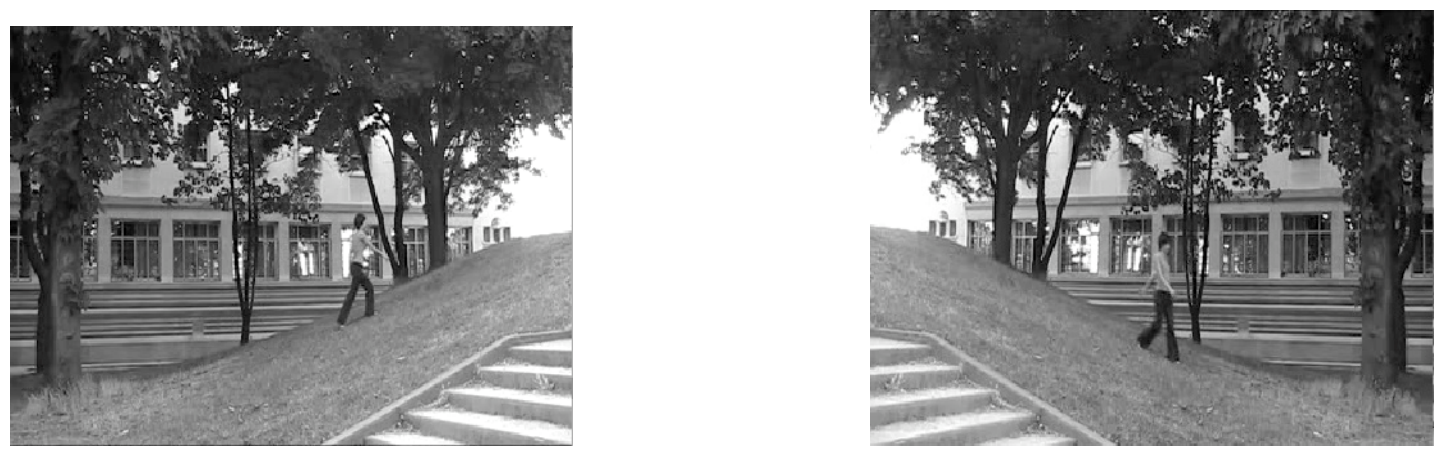

Exemple 8 : « on voit quelqu'un qui monte en marchant » $(\mathrm{T}+\mathrm{M})$

La consigne indiquée au participant est la suivante : «Vous allez entendre une phrase et en même temps vous allez voir deux vidéos, une à gauche et une à droite. Vous allez devoir choisir celle des deux vidéos, à gauche ou à droite, qui correspond à la phrase entendue. »

Nous avons utilisé le système CHILDES (Child Language Data Exchange System) pour la transcription et l'analyse des données, la transcription ayant été réalisée conformément aux règles CHAT (Codes for the Human Analysis of Transcripts) et avec l'aide du logiciel CLAN (Computerized Language Analysis) et du système de codage spécifique (Hickmann et al., 2009) adapté pour les besoins de la présente étude.

\section{Participants}

Deux patients aphasiques ont participé à l'étude (Boudre, 2009). Ils ont bénéficié d'une évaluation clinique préalable visant à écarter des troubles de compréhension et d'analyse visuelle susceptibles de compromettre la réalisation des épreuves (difficultés de compréhension des consignes ou d'appréhension des vidéos ou animations). L'évaluation du langage (BDAE, Mazaux \& Orgogozo, 1982 et DO80, Deloche \& Hannequin, 1997) a permis de confirmer deux profils (aphasie non fluente avec troubles de la syntaxe prédominants et aphasie fluente avec troubles lexicaux).

Le premier sujet, MD, était un patient gaucher âgé de 32 ans, ancien boulanger, victime 27 mois auparavant d'un accident vasculaire hémorragique fronto-pariéto-occipital gauche. Après avoir présenté un tableau initial d'aphasie globale avec hémiplégie droite, celui-ci a évolué vers une aphasie non fluente de type Broca avec une hémiparésie droite pour laquelle un suivi orthophonique plurihebdomadaire était toujours en cours. Au moment où nous avons rencontré MD, ses troubles du langage oral concernaient essentiellement le niveau expressif avec une expression orale réduite, lente et laborieuse avec des troubles arthriques séquellaires (gênant toutefois peu l'intelligibilité de la parole), des troubles lexicaux modérés (DO $80=70 / 80$, seuil pathologique $=72$ ) et un agrammatisme encore important (phrases incomplètes ou structures très simples, omissions de morphèmes flexionnels et de mots grammaticaux).

Le second participant, MP, était un patient droitier, âgé de 76 ans, ancien médecin des armées, victime d'un accident vasculaire ischémique sylvien gauche responsable d'une aphasie anomique. Au moment où nous avons rencontré MD, c'est-à-dire une dizaine de jours après son entrée à l'hôpital, celui-ci s'exprimait de façon fluente, sans troubles syntaxiques mais présentait des troubles lexicaux (hésitations dans le discours, recherches lexicales régulières, production de paraphasies) avec un score de 54/70 au DO 80 (seuil pathologique $=73$ ).

Dans une étude parallèle (Soroli, 2009), le protocole a été proposé à 14 participants sains monolingues de langue maternelle française, comprenant 7 hommes et 7 femmes d'environ 35 ans en moyenne. A partir d'un questionnaire, il a pu être déterminé que ces participants étaient tous droitiers, qu'ils n'avaient jamais été exposés à une langue /culture étrangère pour plus de six mois simultanés, qu'ils n'avaient pas appris une langue étrangère précocement (avant l'âge de 10 ans) et enfin qu'ils n'avaient aucun 
antécédent de troubles neurologiques ou développementaux. Les passations de l'épreuve de production et de compréhension auprès de cette population témoin ont mis en évidence un taux de réussite élevé à l'épreuve de compréhension et, pour l'épreuve de production, une forte focalisation sur la trajectoire, que ce soit pour les mouvements volontaires ou provoqués, conformément à ce qui était attendu pour des locuteurs francophones.

\section{Résultats}

Les résultats se présentent en trois volets : (i) des différences dans le temps nécessaire aux participants sains et aphasiques pour réaliser l'épreuve de compréhension; (ii) un taux de réussite comparable pour cette épreuve ; (iii) des différences dans l'épreuve de production en ce qui concerne le nombre d'informations produites malgré certaines similitudes dans le type d'informations exprimées par les deux populations.

Les résultats à l'épreuve de compréhension figurent dans le Tableau I. Pour ce qui concerne les mesures des temps de réaction l'horloge démarre en même temps que la présentation des stimuli auditifs et visuels et s'arrête au moment de la réponse de la part du sujet. Les temps de réaction sont mesurés en millisecondes (msec). Dans cette tâche nous observons :

(a) un taux de réussite important pour les deux populations avec un léger avantage de la population témoin : les contrôles font en moyenne 3 erreurs sur 74 items (certains allant jusqu'à 8 erreurs) et obtiennent une moyenne de $96 \%$ de réussites. Les aphasiques font chacun 6 erreurs et un taux de $92 \%$ de réussite.

(b) les patients aphasiques mettent environ une fois et demie plus de temps que les contrôles pour réaliser l'épreuve. Les analyses statistiques proposées pour les études de cas (Student test, Crawford \& Garthwaite, 2006) confirment les différences entre les moyennes des scores de chaque patient et celles du groupe contrôle. La moyenne du patient agrammatique en termes de temps de réaction ainsi que celle du patient anomique sont significativement inférieures aux moyennes des scores du groupe contrôle $(\mathrm{t}(13)=12.01, \mathrm{p}<0.0001$, pour l'agrammatique $; \mathrm{t}(13)=33, \mathrm{p}<0.0001$, pour l'anomique).

Tableau I : Taux de réussite et temps de réaction à l'épreuve de compréhension

\begin{tabular}{|c|c|c|}
\hline Groupe & Taux de réussite & Temps de réaction (msc) \\
\hline Contrôles & $96 \%$ & 1793 \\
\hline Agrammatique & $92 \%$ & $2203 * *$ \\
\hline Anomique & $92 \%$ & $3074 * *$ \\
\hline
\end{tabular}

Dans l'épreuve de production, les participants aphasiques se distinguent des contrôles par leurs difficultés expressives : celles-ci se manifestent par une lenteur, de nombreuses pauses (170 et 140 pauses pleines pour chacun des deux corpus) d'expression, des hésitations, des autocorrections et la nécessité d'interventions et d'aides fréquente de la part de l'examinateur pour obtenir un énoncé cible, la production de paraphasies (ex. 11 à 15) pour les deux patients aphasiques et d'énoncés agrammatiques pour MD (ex. 16 et 17: omission du verbe, ex. 18: omission du sujet, ex. 19: absence de flexion verbale), voire phonétiquement incorrects (ex. 20).

(11) « La grenouille sauce [= saute] dans la ... le la » (MD) [item : sauter lac]

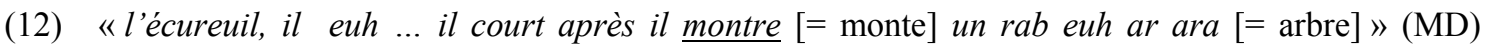
[item : monter arbre - courir]

(13) « le farmier [ $[$ fermier] ramène des pommes » $(\mathrm{MD})$ [item : traverser route - pousser - glisser] 
(14) « ouais un coureur de pion $[=$ fond $]$... un coureur de fond » (MP) [item : traverser route - courir $]$

(15) «ben il escabale $[=$ escalade $]$... escalade le nain $[=$ le nid $]$... le nid » (MP) [item : monter poteau courir]

(16) « une fille trottinette [= fait de la trottinette] il euh, une ... une fille euh, trottinette euh euh, la porte ... juste [= jusqu' à] la porte » (MD) [item : entrer maison - trottinette ]

(17) « euh ... ben porte $»(\mathrm{MD})$ [item : entrer maison - marcher ]

(18) « marche...euh... quatre pattes » (MD) [item : traverser rue - à quatre pattes ]

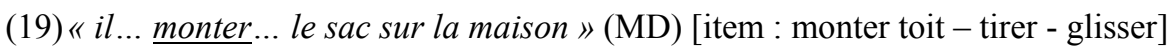

(20) « il euh prend une banane, et il descend le arbre» (MD) [item : descendre arbre - glisser]

Le tableau II montre la proportion d'énoncés en fonction de leur densité sémantique. La densité sémantique (DS) permet de rendre compte de la richesse informationnelle des énoncés produits. Ce terme désigne le nombre de composantes sémantiques concernant le mouvement (trajectoire, manière du déplacement de l'objet ou de l'agent, manière de la cause) présents dans l'énoncé. Par définition, les mouvements provoqués sont susceptibles de donner lieu à des énoncés comprenant davantage d'informations (DS1, DS2 ou DS3) que les mouvements volontaires (DS1 ou DS2).

Les résultats indiquent que les énoncés produits par le groupe contrôle sont plus riches que ceux produits par les patients aphasiques. En moyennant la densité totale des réponses, les différences entre chaque patient et le groupe contrôle sont significatives, aussi bien pour les mouvements volontaires (items situés d'animations : $\mathrm{t}(13)=4.26, \mathrm{p}<0.001$, items vidéos situés : $\mathrm{t}(13)=3.66, \mathrm{p}<0.002$, pour l'agrammatique et $\mathrm{t}(13)=2.11, \mathrm{p}<0.03$ pour l'anomique, items non situés : $\mathrm{t}(13)=2.70, \mathrm{p}<0.01)$ que pour les mouvements provoqués $(\mathrm{t}(13)=4.62, \mathrm{p}<0.0001)$. En effet, le groupe contrôle produit majoritairement des énoncés de densité sémantique 2 et 3 , alors que les patients produisent majoritairement des énoncés de densité sémantique 1.

Tableau II : Densité sémantique des réponses

\begin{tabular}{|c|c|c|c|}
\hline \multicolumn{4}{|c|}{ Mouvements Volontaires I (animations) } \\
\hline & Contrôles & Agrammatique & Anomique \\
\hline DS0 & $4 \%$ & $6 \%$ ** & $11 \%$ ** \\
\hline DS1 & $58 \%$ & $72 \%$ ** & $67 \%$ ** \\
\hline DS2 & $38 \%$ & $22 \%$ ** & $22 \%$ ** \\
\hline \multicolumn{4}{|c|}{ Mouvements Volontaires II (vidéos) } \\
\hline & Contrôles & Agrammatique & Anomique \\
\hline DS0 & $1 \%$ & $0 \%$ & $0 \%$ \\
\hline DS1 & $45 \%$ & $70 \%$ ** & $60 \% *$ \\
\hline DS2 & $54 \%$ & $30 \%$ ** & $40 \%{ }^{*}$ \\
\hline \multicolumn{4}{|c|}{ Mouvements Provoqués } \\
\hline & Contrôles & Agrammatique & Anomique \\
\hline DS0 & $2 \%$ & $0 \%$ & $0 \%$ \\
\hline DS1 & $12 \%$ & $50 \%$ ** & $38 \%$ ** \\
\hline DS2 & $63 \%$ & $38 \%$ ** & $63 \%$ \\
\hline DS3 & $23 \%$ & $13 \%$ & $0 \%$ ** \\
\hline
\end{tabular}

$D S n=$ Densité sémantique de l'information spatiale dans la réponse ( $n=$ nombre de composantes exprimées) $* p=0.05-0.01, * * p<0.01$ ou inférieur 
Le tableau III montre les informations spatiales exprimées par les participants dans leurs réponses. Pour les besoins de l'analyse, ces informations ont été traitées de la façon suivante: la Trajectoire ( $T$, pe traverser) ; la Cause exprimée seule $(\mathrm{C}$, mettre $)$; la Manière $(\mathrm{M})$, comprenant la manière du déplacement de l'agent (marcher) ou de l'objet (rouler), ainsi que la manière de l'action effectuée par l'agent pour provoquer le mouvement (pousser).

En ce qui concerne le groupe contrôle, les sujets décrivent les mouvements situés en exprimant la seule Trajectoire (T) ou en la combinant avec la Manière (TM), alors qu'ils décrivent les mouvements non situés en se focalisant sur la seule Manière $(\mathrm{M})$. Avec les mouvements provoqués, le pattern le plus fréquent consiste à exprimer conjointement la Cause et la Manière (CM) mais un nombre substantiel de réponses contiennent les trois composantes (Cause, Manière, Trajectoire : CMT). En ce qui concerne les aphasiques, on constate les deux patterns suivants. Avec les items situés, lorsque les sujets ne donnent qu'une seule information concernant le mouvement, ils privilégient la Trajectoire avec les deux types de mouvements - volontaires et provoqués, exprimant cette composante plus souvent que les contrôles (items animés situés : $\mathrm{t}(13)=2,04, \mathrm{p}<0.05$ pour l'agrammatique, et $\mathrm{t}(13)=1.97, \mathrm{p}<0.05$ pour l'anomique). Avec le mouvement provoqué, on observe un focus sur la Cause exprimée soit seule, soit avec la Trajectoire (CT) $(\mathrm{t}(13)=27, \mathrm{p}<0.0001)$ chez le patient anomique et sur la Trajectoire souvent exprimée seule $(\mathrm{T})$ $(\mathrm{t}(13)=10.69, \mathrm{p}<0.0001)$ et parfois conjointement exprimée avec la Cause (CT) chez le patient agrammatique. Par ailleurs, les deux patients expriment souvent conjointement la Cause et la Trajectoire (CT) $(\mathrm{t}(13)=4.54, \mathrm{p}<0.001)$. Enfin, dans le cas des items non situés, qui ne comportent pas d'informations permettant d'induire de trajectoire (fond d'écran neutre), les aphasiques privilégient la manière, tout comme les contrôles. Néanmoins, alors que la différence entre l'agrammatique et le groupe contrôle n'est significative pour l'expression de la Manière $(\mathrm{t}(13)=1.35, \mathrm{p}>0.05)$, ce patient montre davantage sa préférence pour la trajectoire même pour ces items non situés où on s'attend à des réponses de Manière seule. L'agrammatique continue à exprimer significativement plus de trajectoire $(\mathrm{t}(13)=7.25, \mathrm{p}<0.0001)$ que le groupe contrôle, essayant ainsi de compenser sa difficulté à accéder à la Manière en tant qu'information grammaticalisée. L'anomique, lui, contrairement à l'agrammatique montre un focus significativement plus important sur la Manière par rapport au groupe contrôle $(\mathrm{t}(13)=2.8, \mathrm{p}<0.01)$, information grammaticalisée en Français et ainsi probablement plus accessible pour ce patient. 
Tableau III : Informations spatiales exprimées

\begin{tabular}{|c|c|c|c|}
\hline \multicolumn{4}{|c|}{ Mouvements Volontaires I (animations) } \\
\hline \multicolumn{4}{|c|}{ Items « situés » } \\
\hline & Contrôles & Agrammatique & Anomique \\
\hline TM & $37 \%$ & $22 \%$ ** & $22 \%$ ** \\
\hline $\mathrm{T}$ & $56 \%$ & $67 \%{ }^{*}$ & $50 \% *$ \\
\hline M & $3 \%$ & $6 \%$ ** & $17 \%$ ** \\
\hline NR & $4 \%$ & $6 \%$ ** & $11 \%$ ** \\
\hline \multicolumn{4}{|c|}{ Items « non situés » } \\
\hline & Contrôles & Agrammatique & Anomique \\
\hline TM & $21 \%$ & $0 \%$ ** & $0 \%$ ** \\
\hline $\mathrm{T}$ & $3 \%$ & $13 \% *$ & $0 \% *$ \\
\hline M & $77 \%$ & $88 \%$ & $100 \%$ ** \\
\hline NR & $0 \%$ & $0 \%$ & $0 \%$ \\
\hline \multicolumn{4}{|c|}{ Mouvements Volontaires II (vidéos) } \\
\hline & Contrôles & Agrammatique & Anomique \\
\hline TM & $54 \%$ & $30 \%{ }^{* *}$ & $40 \% *$ \\
\hline $\mathrm{T}$ & $39 \%$ & $30 \%$ & $40 \%$ \\
\hline$M$ & $6 \%$ & $40 \%$ ** & $20 \%$ ** \\
\hline NR & $1 \%$ & $0 \%$ & $0 \%$ \\
\hline \multicolumn{4}{|c|}{ Mouvements Provoqués } \\
\hline & Contrôles & Agrammatique & Anomique \\
\hline CMT & $23 \%$ & $13 \%$ & $0 \%$ ** \\
\hline $\mathrm{CM}$ & $47 \%$ & $0 \%$ ** & $13 \%$ ** \\
\hline $\mathrm{CT}$ & $14 \%$ & $38 \%$ ** & $38 \%$ ** \\
\hline TM & $2 \%$ & $0 \%$ & $13 \%$ \\
\hline $\mathrm{T}$ & $11 \%$ & $50 \%$ ** & $13 \%$ \\
\hline $\mathrm{M}$ & $0 \%$ & $0 \%$ & $0 \%$ \\
\hline C & $1 \%$ & $0 \%$ & $25 \%$ ** \\
\hline NR & $2 \%$ & $0 \%$ & $0 \%$ \\
\hline
\end{tabular}

Enfin, le Tableau IV montre comment les participants répartissent les informations spatiales sur différentes procédures linguistiques dans leurs réponses aux items situés. En particulier, ce tableau distingue l'utilisation du verbe principal et d'autres procédures hors-verbe et/ou à la périphérie de l'énoncé (prépositions, adverbiaux, subordonnées, etc.). En ce qui concerne les mouvements volontaires, le groupe contrôle exprime principalement la seule trajectoire dans le verbe et souvent aucune information hors-verbe. Pour les mouvements provoqués, ces participants utilisent le verbe pour exprimer la Manière - principalement celle de l'action effectuée par l'agent pour provoquer le mouvement - ainsi que la trajectoire, mais expriment très peu d'informations par d'autres moyens. Par comparaison, les patients aphasiques - tout comme les contrôles - décrivent les mouvements volontaires (animations et vidéos) en privilégiant la trajectoire dans le verbe, laissant la manière hors-verbe ou entièrement implicite. Une exception est à noter : le patient agrammatique exprime significativement plus la Manière dans le verbe par opposition aux autres participants, du moins avec les vidéos $(\mathrm{t}(13)=14.67, \mathrm{p}<0.0001)$. Pour ce qui concerne l'expression des mouvements provoqués, les aphasiques utilisent le verbe pour exprimer soit la trajectoire seule (agrammatique: $\mathrm{t}(13)=10.69, \mathrm{p}<0.0001$ ), soit la cause et la trajectoire (anomique: $\mathrm{t}(13)=27, \mathrm{p}<0.0001$ ), sans aucune autre information en dehors du verbe. 
Tableau IV : Répartition de l'information spatiale dans les descriptions des items situés

\begin{tabular}{|c|c|c|c|c|c|c|}
\hline \multicolumn{7}{|c|}{ Mouvements Volontaires I (animations) } \\
\hline & \multicolumn{2}{|c|}{ Contrôles } & \multicolumn{2}{|c|}{ Agrammatique } & \multicolumn{2}{|c|}{ Anomique } \\
\hline & Verbe & Périphérie & Verbe & Périphérie & Verbe & Périphérie \\
\hline TM & $24 \%$ & $2 \%$ & $22 \%{ }^{*}$ & $0 \%$ * & $22 \%$ & $0 \%{ }^{*}$ \\
\hline $\mathrm{T}$ & $70 \%$ & $41 \%$ & $67 \%$ & $6 \%$ ** & $50 \%$ ** & $11 \%$ ** \\
\hline $\mathrm{M}$ & $5 \%$ & $11 \%$ & $6 \%$ & $0 \%$ ** & $17 \%$ ** & $6 \%$ ** \\
\hline NR & $1 \%$ & $46 \%$ & $6 \%$ ** & $94 \%$ ** & $11 \%$ ** & $83 \%$ ** \\
\hline \multicolumn{7}{|c|}{ Mouvements Volontaires II (vidéos) } \\
\hline & \multicolumn{2}{|c|}{ Contrôles } & \multicolumn{2}{|c|}{ Agrammatique } & \multicolumn{2}{|c|}{ Anomique } \\
\hline & Verbe & Périphérie & Verbe & Périphérie & Verbe & Périphérie \\
\hline TM & $26 \%$ & $14 \%$ & $20 \%$ & $0 \%$ ** & $20 \%$ & $0 \% * *$ \\
\hline $\mathrm{T}$ & $59 \%$ & $32 \%$ & $30 \%$ ** & $10 \%$ ** & $50 \%$ & $20 \%$ ** \\
\hline$M$ & $14 \%$ & $16 \%$ & $50 \%$ ** & $0 \%$ ** & $30 \%$ ** & $30 \%$ ** \\
\hline NR & $1 \%$ & $37 \%$ & $0 \%$ & $90 \%$ ** & $0 \%$ & $50 \%$ ** \\
\hline \multicolumn{7}{|c|}{ Mouvements Provoqués } \\
\hline & Contrôles & & Agrammatique & & Anomique & \\
\hline & Verbe & Périphérie & Verbe & Périphérie & Verbe & Périphérie \\
\hline CMT & $12 \%$ & $0 \%$ & $13 \%$ & $0 \%$ & $0 \% *$ & $0 \%$ \\
\hline $\mathrm{CM}$ & $56 \%$ & $3 \%$ & $0 \%$ ** & $0 \% *$ & $13 \%$ ** & $0 \% *$ \\
\hline $\mathrm{CT}$ & $14 \%$ & $0 \%$ & $38 \%$ ** & $0 \%$ & $38 \%$ ** & $0 \%$ \\
\hline TM & $2 \%$ & $0 \%$ & $0 \%$ & $0 \%$ & $13 \%$ & $0 \%$ \\
\hline $\mathrm{T}$ & $11 \%$ & $27 \%$ & $50 \%$ ** & $0 \%$ ** & $13 \%$ & $0 \%$ ** \\
\hline$M$ & $0 \%$ & $0 \%$ & $0 \%$ & $0 \%$ & $0 \%$ & $0 \%$ \\
\hline $\mathrm{C}$ & $1 \%$ & $0 \%$ & $0 \%$ & $0 \%$ & $25 \%$ ** & $0 \%$ \\
\hline NR & $4 \%$ & $71 \%$ & $0 \%$ ** & $0 \%$ ** & $0 \%$ ** & $0 \%$ ** \\
\hline
\end{tabular}

De manière générale, on constate que les participants aphasiques, par rapport aux contrôles, semblent rencontrer des difficultés particulières lorsqu'il s'agit d'associer dans l'énoncé plusieurs informations entre elles (TM, CT, CMT) ce qui apparaît en bonne concordance avec le résultat précédent sur la densité sémantique. MD (agrammatique) et MP (anomique), limités dans leur expression, privilégient certaines informations: le plus souvent la trajectoire (ex. 21 et 22) mais également la manière du déplacement de l'agent lorsque celle-ci est particulièrement saillante dans la vidéo (ex. 23 et 24) mais rarement les deux composantes dans le même énoncé. Le patient agrammatique emploie systématiquement des prépositions simples (ex. 21), omet les prépositions et/ou évite l'énonciation de verbes de manière ayant recours à la périphrase (ex. 22, 23), substitue un morphème par un autre, moins marqué, par exemple le cas nominatif pour les substantifs et l'infinitif pour les verbes (ex. 24 et 25 respectivement) et fait des approximations sémantiques (ex. 26). L'anomique utilise plus souvent des stratégies de compensation lexicale, fournissant systématiquement un mot approchant sémantiquement (ex. 27 et 28) ou ayant recours à la périphrase (ex. 29).

(21) « ... ouais c'est rentre à la grotte» (MD) [item : entrer - pousser - glisser] Recours à la préposition simple (à).

(22) «...la fille sort, euh, euh, euh, euh, la fille sort» (MD) [item : sortir - faire du rollers].

Manière implicite et omission de la préposition.

(23) « ...ouais euh, elle a mis un, des rollers » (MD) [item : entrer - faire du rollers].

Recours à un verbe neutre (mettre), plutôt qu'un verbe indiquant la manière du déplacement (faire du rollers)

(24) « une fille trottinette [= fait de la trottinette] il euh, une ... une fille euh, trottinette euh euh, la porte ... juste [= jusqu' à] la porte » (MD) [item : entrer - trottinette ] 
Omission du verbe de manière de déplacement (faire de la trottinette), remplacement par un nom (trottinette) et approximation lexicale pour compenser la difficulté avec les prépositions (juste au lieu de jusqu’à).

(25) « ...euh, il euh promener » (MD) [item : entrer - marcher].

Substitution du verbe par le morphème le moins marqué ici l'infinitif du verbe se promener (sans le pronom réflexif).

(26) «...il elle sautille tout, euh sur le lac gelé » (MD) [item : traverser lac - faire du patin]

Recours aux approximations sémantiques : sautiller à la place de faire du patin.

(27) « ...elle pédale » (MP) [item : monter colline - vélo]

Recours aux approximations sémantiques avec l'emploi d'un verbe de manière peu habituel pédaler par rapport au plus fréquent faire du vélo, en l'absence de la mention d'un vélo.

(28) «...le petit vieux trimballe une table » (MP) [item : entrer - pousser - glisser].

Recours aux approximations sémantiques.

(29) « ...il marche à pieds joints » (MP) [item : entrer - sauter].

Recours à la périphrase pour compenser le manque du verbe « sauter ».

\section{Discussion}

Cette étude visait à comparer les performances de deux patients aphasiques et celles d'un groupe contrôle dans des tâches de compréhension et de production mettant en scène le mouvement. Les résultats montrent d'abord un bon taux de réussite en compréhension pour les deux populations. Ce résultat indique que les différentes informations étudiées dans le protocole (trajectoire et manière du déplacement, cause et manière de la cause) sont bien traitées par les participants contrôles et aphasiques d'un point de vue réceptif. Malgré leur bonne compréhension d'énoncés spatiaux et une appréhension des notions sousjacentes que l'on peut qualifier de normales, les patients aphasiques prennent significativement plus de temps pour répondre à la tâche. Nous constatons que dans la comparaison statistique des deux cas pathologiques à un groupe de contrôles plus jeune, les temps de réaction du patient anomique de 76 ans qui sont probablement liés à son âge, peuvent paraitre méthodologiquement problématiques. Dans le futur il faudra vérifier ces scores avec des groupes appariés.

Ce n'est que la production qui fait apparaître un certain nombre d'éléments pathologiques : troubles lexicaux (hésitations, lenteur, pauses, recherches lexicales, paraphasies) pour les deux patients (les difficultés de MP étant plus importantes que celles de MD) et syntaxiques pour MD (phrases simples ou incomplètes, rareté des morphèmes grammaticaux : flexions, prépositions, articles, pronoms, omission de certaines parties du discours...).

Les difficultés linguistiques rencontrées par les participants aphasiques les conduisent à privilégier certaines informations, à aller vers ce qui leur est le plus accessible et à simplifier leur expression afin de réaliser la tâche de description. Dans l'analyse du focus, nous constatons une préférence globale pour la composante Trajectoire lorsqu'elle est exprimée seule, et ceci dans tout les types d'items situés. Nous constatons aussi une préférence pour la Trajectoire dans le cas du patient agrammatique qui se focalise sur l'information typiquement lexicalisée étant donnée son déficit grammatical. En revanche, l'anomique exprime la Cause, parfois conjointement avec la Trajectoire et parfois seule. Utilisant principalement le verbe. Ce résultat va à l'encontre de nos attentes puisque la Cause est typiquement exprimée par les contrôles dans la périphérie du verbe (subordonnée). Il devra donc être examiné dans des recherches futures. Ce comportement s'accompagne d'une densité sémantique moindre et par des énoncés minimaux ou plus pauvres montrant la difficulté que rencontrent les participants aphasiques à produire conjointement plusieurs informations dans un même énoncé. Par exemple, lorsqu'il il s'agit de décrire les mouvements volontaires, les participants contrôles expriment souvent la seule Trajectoire (T), mais allient également la Trajectoire et la Manière (TM), alors que les patients aphasiques se trouvent contraints d'exprimer la plupart du temps une seule information, selon le type d'item proposé et leur type de déficit. Lorsque la trajectoire et la manière sont en concurrence, les participants aphasiques de notre étude ont tendance à se focaliser généralement sur la trajectoire alors que l'expression de la manière est 
potentiellement disponible (cf. résultats aux items non situés). Ceci confirme aussi le caractère saillant de la Trajectoire, information de base exprimée dans toutes les langues, par rapport à la Manière.

Par ailleurs, nous observons le développement des stratégies de compensation vers d'autres patterns plus accessibles. L'anomique se focalise sur la composante Cause avec le mouvement provoqué, et l'agrammatique exprime systématiquement la Trajectoire seule ou la Cause et la Trajectoire conjointes, lorsque les items présentés le lui permettent. Ce faisant, il semble que les aphasiques privilégient une information qui est plus facilement encodée par la langue française, chacun par rapport à son déficit. En ce qui concerne la composante Trajectoire, les énoncés des participants aphasiques sont comparables à ceux des participants contrôles avec une expression préférentielle de la trajectoire dans le verbe principal. On peut ainsi dire que, si les troubles aphasiques présentés par MD et MP sont à l'origine d'une réduction de la quantité d'informations spatiales exprimées dans leurs énoncés, la grande majorité de leurs réponses ne modifie pas l'organisation des informations Manière et Trajectoire propre à la langue française.

La comparaison des résultats de nos deux populations fait donc apparaître des différences dans l'expression du mouvement qui sont liées à la pathologie aphasique, mais aussi des similitudes qui peuvent être attribuée à la structure de la langue commune parlée par tous les locuteurs ayant participé à l'expérimentation (contrôles et aphasiques). De ce point de vue, nous poursuivons actuellement ces recherches avec plusieurs types de participants : d'autres participants aphasiques monolingues de langue française, aussi bien agrammatiques qu'anomiques; des patients d'autres langues dont les résultats devront être confrontés à ceux des études translinguistiques chez les participants sans troubles (cf. Soroli, en préparation), mais aussi des patients bilingues (cf. l'étude de N'guyen 2007, utilisant un protocole similaire sur un patient aphasique bilingue français-anglais).

\section{Conclusion}

Les résultats de notre étude sur l'expression du mouvement en français et en aphasie sont de nature à conforter la classification de Talmy $(1985,2000)$ qui décrit le français comme une langue à cadrage verbal, c'est-à-dire, une langue qui privilégie l'expression de la trajectoire dans le verbe et de la manière en périphérie. Ils confirment un fait qui était prévisible: l'aphasie vient modifier l'expression du mouvement. Les énoncés des participants aphasiques sont plus pauvres que ceux des contrôles, que ce soit pour les mouvements volontaires ou pour les mouvements provoqués. Cette différence peut être interprétée comme une difficulté particulière des participants aphasiques, en raison de leurs troubles du langage, à associer dans un même énoncé plusieurs informations, que celles-ci concernent la Trajectoire et la Manière du déplacement ou qu'elles aient trait à sa Cause. En effet, cette expression conjointe constitue un coût cognitif supplémentaire que les patients ont du mal à réaliser. Leur stratégie consiste alors à aller vers ce qui est le plus accessible et à utiliser les processus préservés (lexicaux ou syntaxiques selon les cas). Nos résultats nous permettent de préciser le type d'informations privilégiées par nos participants aphasiques : il s'agit de l'information concernant la Trajectoire correspondant à l'information préférentiellement encodée en français et notamment lexicalisée par le verbe. Cette étude ne concerne cependant que deux participants aphasiques, d'âges différents (un patient jeune et un patient âgé), à des distances différentes de la lésion (à la phase initiale et à distance de la lésion), et doit à ce titre être considérée comme exploratoire dans le cadre plus large de travaux en cours concernant la représentation spatiale dans une perspective translinguistique en aphasie. 


\section{Références bibliographiques}

Boudre, H. (2009). Incidences des troubles lexicaux et syntaxiques sur l'expression du mouvement chez l'aphasique : étude de deux cas. Mémoire d'Orthophonie. Université de Lille 2.

Bowerman, M. (1996). The origins of children's spatial semantic categories: Cognitive versus linguistic determinants. In Gumperz, J.J. \& Levinson, S.C. (Eds.). Rethinking linguistic relativity. Cambridge University Press, 145-176.

Bowerman, M. (2007). Containment, support, and beyond: Constructing topological spatial categories in first language acquisition. In Aurnague, M., Hickmann, M. \& Vieu, L. (Eds.). The categorization of Spatial Entities of Language and Cognition. Amsterdam : John Benjamins, 177-203.

Hickmann, H. Hendriks, H. Demagny, A-C. Engelman, H., Iakovleva, T. M., Ji Yinglin. Ochsenbauer, A-K. \& Soroli, E. (2009). Etudes expérimentales de la représentation de l'espace: Manuel de codage, Laboratoire Structures Formelles du Langage. CNRS \& Université de Paris 8.

Choi, S. \& Bowerman, M. (1991). Learning to express motion events in English and Korean: the influence of languagespecific lexicalization patterns. Cognition 41, 83-121.

Deloche, G. \& Hannequin, D. (1997). DO 80 : Test de dénomination orale d'images. Issy-les-Moulineaux : Editions ECPA.

Fayol, M. (1997). Des idées au texte, psychologie cognitive de la production verbale, orale et écrite. Paris : PUF.

Goodglass, H. \& Gleason, JB. (1991). Introduction. Brain and language 41 (2. Special Issue: Crosslinguistic Studies of Aphasia), 121-122.

Hickmann M. (2003). Children's discourse: Person, space and time across languages. Cambridge: Cambridge niversity Press.

Hickmann, M. (2006). The relativity of motion in first language acquisition in Hickmann M. \& Robert S. (Eds.), Space across languages : linguistic systems and cognitive categories. Amsterdam : Benjamins, 281-308.

Hickmann, M. (2007). Static and dynamic location in French: Developmental and cross-linguistic perspectives, in Aurnague, M. Hickmann, M. \& Vieu, M. (Eds). The categorization of Spatial Entities of Language and Cognition. John Benjamins Publishing Compagny, 205-231.

Hickmann, M. Hendriks \& H., Demagny, A-C. (2008). How English learners express caused motion in French. AILE 27, $15-41$.

Hickmann, M., Tarrane, P. \& Bonnet, Ph. (2009). Motion in first language acquisition: manner and path in French and in English. Journal of Child Language, 36:4, 705-741.

Hickmann, M. \& Nespoulous J-L. (non publié). Syntaxe, lexique et représentation de l'espace: perspectives translinguistiques sur l'aphasie.

Kopecka, A. (2004). Etude typologique de l'expression de l'espace : localisation et déplacement en français et en polonais. Thèse de doctorat. Université Lumière Lyon 2.

Kopecka, A. (2006). The semantic structure of motion verbs in French : Typological perspectives. In Hickmann M. \& Robert S. (Eds.). Space across languages: Linguistic systems and cognitive categories. Amsterdam: John Benjamins, 83-101. 
Kremin, H. (1994). Altérations spécifiques des composantes du langage. 2. Perturbations lexicales : les troubles de la dénomination. In Seron, X. et Jeannerod, M. Neuropsychologie Humaine. Bruxelles : Mardaga, 375-389.

Lemmens, M. (2005). De la sémantique lexicale à la typologie sémantique : vers une sémantique basée sur l'activité langagière. Habilitation à diriger des recherches. Université de Lille 3.

Levinson, S. C. (1996). Relativity in spatial conception and description. In Gumperz, J.J. \& Levinson, S.C. (Eds.), Rethinking linguistic relativity. Cambridge : Cambridge University Press, 177-202.

Mazaux, J.-M., Orgogozo, J.-M. (1982). Échelle d'évaluation de l'aphasie d'après: Boston Diagnostic Aphasia Examination (Goodglass et Kaplan, 1972). Issy les Moulineaux : ECPA.

Menn, L. \& Obler L.K. (1990). Language data and theories of agrammatism. In Menn, L.\& Obler, L.K. (Eds.), Agrammatic Aphasia. A cross language narrative sourcebook, Amsterdam: John Benjamins Publishing Company.

N'Guyen, H. (2007). L'aphasie des bilingues : une étude translinguistique de l'expression du mouvement à travers les productions d'une personne aphasique bilingue anglais/français. Mémoire d'orthophonie. Université de Lille II.

Nespoulous, J-L. (1999). Universal vs language-specific constraints in agrammatic aphasia. In Fuchs, C.\& Robert, S. (Eds.) Language diversity and cognitive representations. John Benjamins, 195-207.

Pillon, A. \& Nespoulous, J-L. (1994), Altérations spécifiques des composantes du langage. 3. Perturbations syntaxiques dans le langage aphasique. In Jeannerod, M. \& Seron, X. (Eds.) Neuropsychologie Humaine. Bruxelles : Mardaga, 390-407.

Segui, J. \& Ferrand, L. (2000). Leçons de parole. Paris : Odile Jacob.

Slobin, D.I. (1996). Two ways to travel: Verbs of motion in English and Spanish. In Shibatani, M. \& Thompson, S.A. (éds), Grammatical constructions: Their form and meaning. Oxford : Oxford University Press, 195-217.

Slobin, D. I. (2004). The many ways to search for a frog: linguistic typology and the expression of motion events. In Strömqvist, S. \& Verhoeven L. (Eds). Relating Events in Narrative. Vol 2, 219-257. Mahwah, NJ: LEA.

Slobin, D. I.. (2006). What makes manner of motion salient? - Explorations in linguistic typology, discourse and cognition. In M. Hickmann \& S. Robert (Eds.). Space across languages : Linguistic systems and cognitive categories. Amsterdam : John Benjamins, 59-81.

Soroli, E. Boudre, H., Hickmann M., Nespoulous J-L. \& Tran, T.M. (2009). Spatial language and cognition: crosslinguistic perspectives in aphasia. Communication orale. The Science of Aphasia International Conference. Antalya, 29 septembre-2 octobre 2009.

Soroli, E. (en préparation). Langage et Cognition spatiale en français, en anglais et en grec: perspectives translinguistiques en aphasie. Thèse de doctorat. Université Paris 8.

Talmy, L. (1985). Lexicalisation pattern : Semantic structure in lexical forms. In Shopen T. (Ed.), Language typology and lexical description, vol. 3, Grammatical categories and the lexicon. Cambridge : Cambridge university Press, 36-146.

Talmy, L. (2000). Towards a cognitive semantics. Volume 1: Concept structuring systems. Volume 2: Typology and process in concept structuring. Cambridge, MA: MIT Press. 